Research Article

\title{
Tennis Rehabilitation Training-Assisted Paclitaxel Nanoparticles in Treatment of Lung Tumor
}

\author{
Di Hu ${ }^{1}$ and YiMin Yang ${ }^{2}$ \\ ${ }^{1}$ School of Sports Institute, Wuhan Business University, Wuhan 430056, Hubei, China \\ ${ }^{2}$ School of International Tennis Academy, Wuhan Sports University, Wuhan 430079, Hubei, China \\ Correspondence should be addressed to Di Hu; ddpp916@163.com
}

Received 18 September 2020; Revised 23 October 2020; Accepted 26 October 2020; Published 20 November 2020

Academic Editor: Tifeng Jiao

Copyright @ 2020 Di Hu and YiMin Yang. This is an open access article distributed under the Creative Commons Attribution License, which permits unrestricted use, distribution, and reproduction in any medium, provided the original work is properly cited.

\begin{abstract}
Paclitaxel nanoparticles are a compound with unique anticancer effects. Its mechanism of action is to prevent tumor rupture by stabilizing tumor proteins, while preventing cell division, leading to cell death, thereby inhibiting the spread of lung tumors. This article aims to study the treatment of lung tumors with paclitaxel nanoparticles assisted by tennis rehabilitation training. In this paper, paclitaxel nanoparticles were prepared by a solvent displacement method, and their particle size and morphology were measured. The TA2 series of experimental rats were selected to establish animal lung tumor models, and they were randomly divided into 5 groups: local injection of saline, porphyrin, and low-, medium-, and high-dose paclitaxel nanoparticles for treatment. The experimental results in this paper show that the average particle size of the paclitaxel nanoparticles prepared in the experiment is about $153,54 \mathrm{~nm}$. Each treatment group inhibited tumor development to varying degrees. Among them, the inhibitory volume rate of paclitaxel nanoparticles in the middle- and high-dose groups was significantly higher than that in the paclitaxel treatment group, indicating that paclitaxel nanoparticles can release drugs slowly.
\end{abstract}

\section{Introduction}

From the 1970s to the 1980s, Granqvist, Buhrman, and others first proposed the term nanoparticle in the United States and conducted in-depth theoretical research. The term "nanoparticle" is closely related to modern science. But it has a long history in real life. In nanoscience, a particle is defined as an independent unit that can describe the properties of matter. There are many studies on nanoparticles in the scientific community today. This is because nanoparticles have many possible applications in the fields of medicine, physics, optics, and electronics. Many countries and institutions, including my country and the National Institute of Nanotechnology in the United States, have provided a lot of government funds to concentrate on studying different types of nanoparticles through experiments, theories, and computer simulations.

Tumors in society are now one of the main factors affecting human health. As the main application of nanoparticles as a drug container, it is very important for people to fully understand the interaction between nanoparticles and biofilms. In the past ten years, due to the wide application of nanoparticles in drug delivery, therapy, and bioimaging, their interaction with cell membranes has attracted more and more attention, but their safety issues have also attracted much attention. Understanding the basic principles of the interaction between nanoparticles and cell membranes is important for improving the evaluation of nanoparticles and their potential toxicity.

Noveletto et al.'s new methods of sports rehabilitation include serious games because they include (in a motivating way) the three basic elements of rehabilitation: reinforcement, repetition, and task-oriented training. His research aims to evaluate the therapeutic effects of biomedical SG and scoring system for lower limb exercise rehabilitation of patients with hemiplegic stroke. He was inspired by the classic video game Pong, whose goal is to not only to control a tennis racket but also to use muscle power. However, there are too many uncontrollable factors in tennis rackets, which 
will make muscle training not so smooth [1]. The purpose of this study by $\mathrm{Yu}$ et al. is to explore the synergistic antitumor effect and mechanism of paclitaxel nanoparticles (PTX-NPs) combined with radiotherapy (RT) on human cervical cancer. Using methoxy poly(ethylene glycol)-poly to prepare PTXNP by solid-phase dispersion method and combining with RT can effectively treat cervical cancer. The in vivo antitumor activity of PTX-NP combined with RT was evaluated using nude mice carrying Hela cell xenograft tumors. The results were evaluated using microfluorine 18-deoxyglucose PET/ computed tomography and immunohistochemistry. However, due to the unstable factors of mixed chemicals, the result data will be inaccurate [2]. Rozario et al. have developed a novel method to track lung tumor movement during radiotherapy in real time. The goal is to gradually increase the target radiation dose while reducing the dose to sensitive structures, thereby increasing local control without increasing toxicity. This method uses the analog and digital reconstructed radiographs as a reference to analyze the megavolt image of the eye diagram of the beam for radiotherapy treatment, but the technology of tracking motion is too complicated and not so easy to implement [3].

The innovation of this article lies in the application of nanotechnology in photodynamic tumor therapy, which can improve the stability of photosensitizers, volume targeting, the depth of action of photodynamic therapy, and simple oxygen production in various ways. Based on nanoparticles, photodynamic therapy can also be combined with other therapies. The application of nanotechnology to photodynamic therapy can improve the effect of tumor treatment, reduce adverse effects, and expand the scope. This is a new technology with great development potential.

\section{Tennis Rehabilitation Training-Assisted Nanoparticle Method}

2.1. Liquid Chromatography. The absorption efficiency of paclitaxel nanoparticles was determined by high-performance liquid chromatography. Accurately weigh about $2 \mathrm{mg}$ of nanoparticles, dissolve them in $5 \mathrm{~mL}$ of acetonitrile, add distilled water to make the volume $10 \mathrm{~mL}$, stir, and filter thoroughly. As a sample solution, the point external standard method was used for the determination, and the external standard was paclitaxel. The HPLC experimental conditions were $\mathrm{C} 18$ column reversed phase; mobile phase was acetonitrile-water (volume ratio $40: 40$ ): flow rate was $1.2 \mathrm{~mL} / \mathrm{min}$; and detection wavelength was $234 \mathrm{~nm}$ [4].

\subsection{Nanoparticle Preparation Method}

2.2.1. Solvent Replacement Method. The displacement of the thermodynamically stable dispersion system formed by the miscible liquid is called the solvent displacement method and is used for the manufacturing magnetic nanoparticles. By changing the ratio of alcohol, oil, and water in the reaction system, the morphology and particle diameter of the reaction system can be changed. In the reaction system, hydrophilic groups and hydrophobic groups can not only dissolve and disperse nanoparticles but also prevent the accumulation of nanoparticles. This paper studies the ultrathin silica particles produced by the hydrolysis of tetraethyltetracycline by the microcoating method and shows that the dependent particle size of the compound is determined by the linear water-oxygen ratio and the concentration of ammonium hydroxide. The nanoparticles prepared by the microalgae method have good morphology and small particle size [5]. However, due to the complexity of the reaction system, the introduction of organic solvents, the control of the reaction time, etc., the processing after the experiment becomes complicated.

2.2.2. Hydrothermal Synthesis Method. The hydrothermal method is a synthetic method that uses substances in an aqueous solution to undergo chemical reactions under high temperature and pressure. It has developed rapidly, among which American researchers used hydrothermal synthesis to make the crystal framework, which laid a good foundation for the rapid development of hydrothermal synthesis [6]. The other one used water, ethyl orthosilicate, triethanolamine, and tetraethylammonium hydroxide as raw materials and synthesized a new type of intermediate silicate hydrothermal composition in a certain proportion and method. It is mainly used in catalytic reactions.

The heat of solution method makes up for some of the shortcomings of the hydrothermal method. Its production principle is similar to that of the hydrothermal method. It changes the water solvent into a solvent. For example, the hydrothermal method is not suitable for easily hydrolyzed compounds, which also expands the field of material production [7].

2.2.3. Precipitation Method. The method of depositing inorganic substances on the nanocore particles and forming a shell layer on the surface of the inorganic substances after hydrolysis is called the precipitation method. During the reaction, in order to deposit inorganic materials on the surface of the nanoparticles, the coupling coefficient needs to be increased. In this paper, bipolar nonionic polymer is used as the coupling agent, and ethyl silicate is used to make polyvinylpyrrolidone adsorb on the surface of nanoparticles. After adding ethyl orthosilicate to the solution for hydrolysis, the nanoparticles with silica shell on the surface are taken to prepare composite nanomaterials [8].

2.2.4. Sol-Gel Method. In the sol-gel method, the raw materials are first dispersed in a solvent. If a reactive monomer is generated by the hydrolysis reaction, the reactive monomer will polymerize to form a salt, forming a macroscopically restricted network structure. Finally, the target material is prepared by drying and heat treatment. The solgel method is a widely used method. The preparation conditions are mild, and the reaction process control is simple. The main chemical reactions are hydrolysis and concentration. However, the gel-sol method has developed 
rapidly due to its long-term reaction and perishable substances.

\subsection{Motion Flow Evaluation Algorithm for Sports Rehabili-} tation Training. To analyze the athlete's movement information, it is necessary to record the three-dimensional coordinate values of the connection points received from Kinect in real time. The infrared camera of the motion detector can reach a maximum rate of 30 frames per second. At this rate, the system will record a maximum of 450 image frames within the specified training time range, that is, the spatial coordinate values of maximum 450 connection points. The three-dimensional point set created in this way is used as a set of training data. If the standard energy is input, in order to achieve the distance between the two point sets, it can be used as an evaluation criterion for an appropriate driving route [9]. This paper uses Hausdorff distance algorithm and time dynamic distortion algorithm to match the joint space coordinate set and template coordinate set and combines the efficiency and error of the two algorithms to calculate the final motion similarity value. This method is combined with the action H-D similarity evaluation method, and two ranging algorithms are combined. The specific rules are as follows:

$$
G=\left[\alpha G_{\mathrm{HD}}+(1-\alpha) G_{\mathrm{DTW}}\right] .
$$

Among them, $D_{\mathrm{HD}}$ and $G_{\mathrm{DTW}}$ are the similarities between the training action flow calculated by the Hausdorff distance measurement algorithm and the time distortion dynamic algorithm. In this article, it is represented from level 1 to level 5; $\alpha$ represents the confidence level of the Hausdorff distance measurement algorithm in the evaluation result. On the contrary, the confidence of the evaluation result of the latent time distortion algorithm is represented by $1-\alpha$. $G$ is the final equivalent score of the training action process and the standard action process, which is the result of rounding the trust of the two algorithms [10].

Rehabilitation training conforms to the characteristics of periodic training. This article introduces a method of evaluating the similarity between periods of approximate periodic signals for the traditional Chinese massage technique to evaluate the similarity of the changes in the action angle waveform.

The matching problem of two time series can be solved according to the inconsistency of the period, and the two similar periodic signal sequences are as follows:

$$
\begin{aligned}
T_{i} & =\left\{s_{i}, s_{i+1}, \ldots, s_{t_{i}+n_{i}-1}\right\}, \\
T_{j} & =\left\{s_{j}, s_{j+1}, \ldots, s_{t_{j}+n_{j}-1}\right\} .
\end{aligned}
$$

The sequence length is represented by $n_{i}$ and $n_{j}$, respectively. According to the basic principle of linear interpolation, the $T_{j}$ sequence is compressed to the same as the $T_{i}$ sequence, and this new sequence is marked as $\left\{T_{j}^{\prime}\right\}$. The waveform error is

$$
e_{i, j}=\frac{\sum_{k=1}^{n_{i}}\left|p_{i, k}-p_{j, k}\right|}{n_{i}}
$$

Then, $\left\{T_{i}\right\}$ the average waveform error for other subsequences is

$$
\overline{e_{i}}=\frac{1}{m-2} \sum_{k=1}^{m-1} e_{i, k}
$$

Among them,

$$
\bar{s}=\frac{1}{m-1} \sum_{k=1}^{m-1}\left(\max _{1 \leq k \leq m-1}\left\{T_{k}\right\}-\min _{1 \leq k \leq m-1}\left\{T_{k}\right\}\right) .
$$

The above formula represents the average value of the maximum change in subsequence information. The similarity of $S$ waves shows that, for the average error of consecutive periodic signal sequences, if the error is smaller, the two shapes are more similar, and the greater the error, the lower the similarity [11].

\section{Paclitaxel Nanoparticles Treatment of Lung Tumor Experiment}

\subsection{Preparation of Paclitaxel Nanoparticles}

\subsubsection{Experimental Steps}

Step 1: dissolve hydrophobic anticancer drug molecules in a good solvent to make hydrophobic anticancer drug molecules, prepare a hydrophobic anticancer drug molecule solution according to the volume ratio of good solvent to poor solvent (0.5-5): 100. The anticancer molecule solution was added to a poor solvent at a temperature of $20-30^{\circ} \mathrm{C}$, magnetically stirred for 1-120 minutes, and then frozen to obtain a preliminary nanoparticle suspension for milking [12].

Step 2: add an aqueous solution of amphibian thiazine with a concentration of $1-10 \mathrm{mg} / \mathrm{mL}$ to the nanoparticle presuspension with a volume ratio of $1 / 100$. After ultrasonic dispersion for 1-120 minutes, you can take the nanometer containing anticancer drugs. Particles and nanoparticles containing anticancer drugs may be dispersed in the water phase.

3.1.2. Experimental Preparation. The composition of medical polycaprolactone adopts vacuum distillation to perfect the monomer caprolactone. Under the protection of nitrogen, the catalyst F68 was added to the polymerization tube in proportion, and octane was added for proportionality. Evacuate and fill with high-purity nitrogen, replace it three times, and then seal the pipeline under vacuum [13]. After mixing the reagents uniformly in a 140 watt oil bath, they are transported to a constant temperature kiln at $140^{\circ} \mathrm{C}$ for 48 hours and then discharged for cleaning.

Accurately weigh polycaprolactone paclitaxel powder and dissolve it in acetone solution. After the stirring state $(120 \mathrm{r} / \mathrm{min})$ is completely dissolved, slowly inject the acetone-paclitaxel-polycaprolactone solution into the polyvinyl 
alcohol aqueous solution, emulsify it with a stirrer to form an oil-water emulsion, and stir in the absorber under normal pressure. Until the organic solvent is completely removed, it is centrifuged at $23000 \mathrm{r} / \mathrm{min}$ for 30 minutes, washed and centrifuged three times, then precipitated with distilled water to remove free paclitaxel and polyvinyl alcohol, and finally stored in the frozen state. Collect all hypertexts at the same time during centrifugation and washing to detect the contents of free packages [14].

\subsection{Preparation Experiment of Lung Tumor Cells}

3.2.1. Cell Recovery. Before resuscitating the cells, use a sterilized ultraclean UV lamp stand for 30 minutes and wipe with $75 \%$ ethanol. Then, quickly remove the previously frozen lung cancer cells from the nitrogen tank and transfer them to the water pipe in the tank at $37^{\circ} \mathrm{C}$. Stir gently to thaw the frozen solution quickly. First spray $75 \%$ alcohol into the cooling chamber and then move it to the aseptic workbench for operation. Before adding the appropriate amount of medium, put the frozen cells in a centrifuge tube, centrifuge at $1000 \mathrm{rpm}$, remove the hypertext, add fresh medium with a small size, add evenly, and resuspend the cells. Transfer the suspended cell liquid to a Petri dish containing $3 \mathrm{ml}$ of fresh medium, and place it in a $37^{\circ} \mathrm{C}$ stable temperature incubator with $5 \%$ carbon for cell culture [15].

3.2.2. Cell Passage. Lung tumor cells are attached cells, so cell culture is carried out in the usual way. The cell density reached $80 \%$ of the lower surface of the dermatophyte and considered to be able to pass down. The workbench after sterilization should be sterilized by ultraviolet radiation 30 minutes in advance, and a tube used on the workbench should be wiped with $75 \%$ ethanol. Gently push the surface with a pistol, then drip the old medium, and then rinse the cell surface with $1 \mathrm{ml}$ of PBS. Repeat the same function three times [16]. After washing with PBS, add $1 \mathrm{ml}$ of coagulation to stop the cloth and make the cloth completely contact the cells. After stopping the drug for 2 minutes, transfer to an inverted microscope to observe cell inhibition. When the cells become round and the cell spacing increases, discard tryptophan, add $2 \mathrm{ml}$ of fresh medium to stop the digestion, slowly and repeatedly press to wash the cells attached to the crop plate, then transfer the cell fluid to a $10 \mathrm{ml}$ centrifuge tube, and centrifuge at $1000 \mathrm{rpm}$. After centrifugation, remove the hypertext, add an appropriate amount of fresh medium, press the cells carefully with a pipette about 7 times until the cell fluid is evenly dispersed, and then place the cell fluid in a new Petri dish to continue cultivation for future use [17].

3.3. Experiments of Paclitaxel Nanoparticles in the Treatment of Lung Tumors. Fifty lung tumor mice were randomly divided into 5 groups: blank control group, paclitaxel positive control group, and low-, medium-, and high-dose paclitaxel nanoparticle groups, with 10 mice in each group. The positive control group was treated with purpurin on the 8th and 11th days. On the 14th and 17th days after tumor vaccine inoculation, the paclitaxel nanoparticle group was given only on the 8th day after inoculation (low-dose group $30 \mathrm{mg} / \mathrm{kg}, 60 \mathrm{mg} / \mathrm{kg}$ in the medium-dose group, and $90 \mathrm{mg} / \mathrm{kg}$ in the high-dose group (local injection); blank control group: no intervention was done. Starting from day 0 , the volume is measured every two days. Flow cytometry measures cell cycle changes [18].

Main content: pathological observation and evaluation of the weight, size, and cell flow of experimental mice before and after treatment on the therapeutic effect of experimental mice with lung cancer were performed.

\section{Analysis of Paclitaxel Nanoparticles in the Treatment of Lung Tumors}

\subsection{Paclitaxel Nanoparticle Analysis}

4.1.1. In Vitro Characterization Analysis. In vitro characterization of paclitaxel nanoparticles measured an average particle size of $234.53 \mathrm{~nm}$ and a distribution coefficient of 0.136 , as shown in Figure 1. The particles were observed by transmission electron microscope as spherical objects of uniform size, and the diameter size was consistent with the particle size detection results. Experimentally, the drug content of paclitaxel nanoparticles was $19.58 \%$, and the embedding rate was $93.25 \%$ [19].

4.1.2. In Vitro Release Analysis. The in vitro release curve of the particles shows that paclitaxel nanoparticles can continuously release the drug within 30 days, as shown in Figure 2. After testing and calculation, the release rate of the drug in the burst release period is $80 \%$, and the release rate after 30 days is $90 \%$. The release curve of the cumulative release rate versus time shows that the cumulative release curve is close to a straight line, which is basically in line with the constant rate of drug release (level 0 release) kinetics. Paclitaxel nanoparticles can maintain a longer validity period due to the protective effect of the polycaprolactone carrier in the body, can continue to release the drug, and have a significant slow-release effect [20].

4.1.3. Analysis of Inhibition of Lung Tumor Growth. The experiment observed the inhibitory effect of paclitaxel polycaprolactone/F68 blended drug-loaded nanoparticles and paclitaxel injection on the tumor growth of TA2 mouse breast cancer lung metastasis model. From the comparison of tumor growth inhibition rate, it can be seen that the paclitaxel polycaprolactone/F68 blended drug-loaded nanoparticle administration group has a significant effect compared with the blank control group at two doses equivalent to the total paclitaxel amount of 60 and $90 \mathrm{mg} / \mathrm{kg}$. The antitumor effect of the dose group equivalent to $30 \mathrm{mg} / \mathrm{kg}$ of paclitaxel is close to that of the paclitaxel positive control group (the total amount of paclitaxel is $5 \mathrm{mg} / \mathrm{kg} \times 4$ ) [21]. With the increase in the dose of paclitaxel, the antitumor effect of the paclitaxel polycaprolactone/F68 blended drug-loaded nanoparticle administration group also increased. When the total amount of paclitaxel is $90 \mathrm{mg} / \mathrm{kg}$, the antitumor effect of ester/F68 blended drug-loaded 


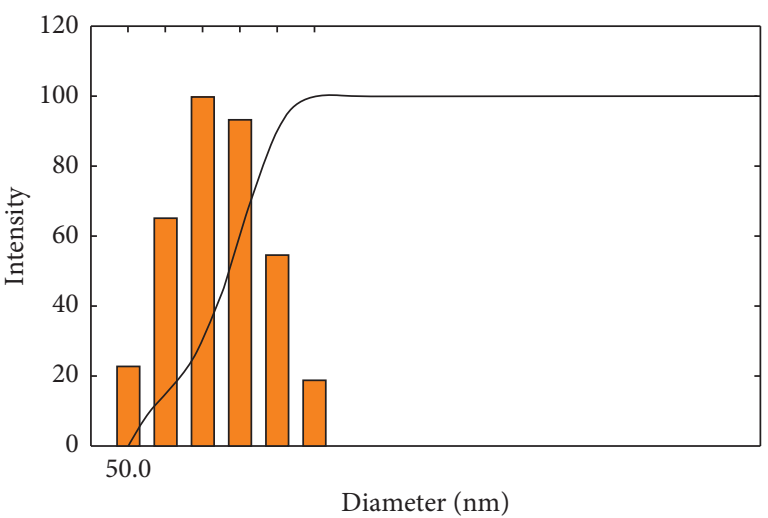

Figure 1: Size distribution of nanoparticles.

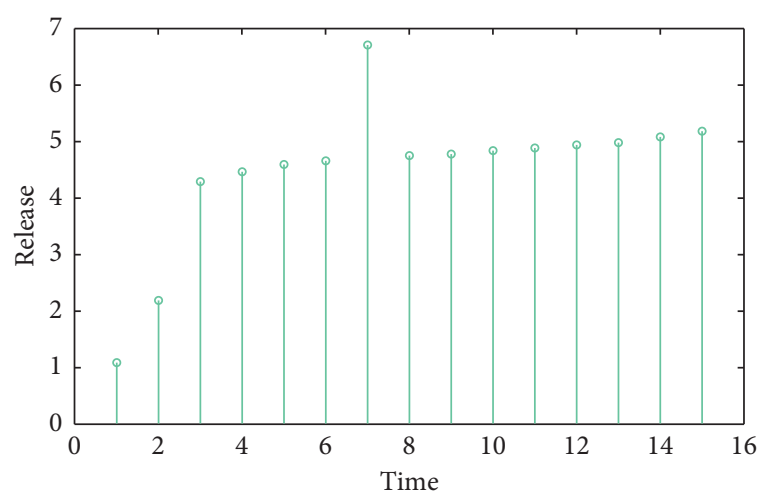

FiguRE 2: In vitro release of paclitaxel nanoparticles.

nanoparticles was significantly higher than that of the paclitaxel injection group. This indicates that the antitumor effect of paclitaxel drug-loaded nanoparticles is dose-dependent [22]. From the observation results of the growth inhibition effect of each administration group on the TA2 mouse breast cancer lung metastasis model, it can be seen that the tumor growth of the mice in the high-dose paclitaxel polycaprolactone/F68 nanoparticle administration group was significantly inhibited, as shown in Figure 3. In the experimental animal group, the tumors of 3 mice disappeared completely.

4.2. Analysis of Paclitaxel Nanoparticles in the Treatment of Lung Tumors. The in vitro activity of PTX and HA preparations was evaluated by the MTT method. The IC50 value calculated by the OD value measured by the MTT method is an important indicator for evaluating the cytotoxicity of drugs or preparations [23]. The smaller the IC50 value, the greater the killing power of the drug on tumor cells.

Table 1 shows the IC50 values of paclitaxel and HA preparations on lung tumor MCF-7 and lung tumor HepG2 cells. It can be seen that the IC50 values of the two cancer cells are relatively small, indicating that the IC50 values for both tumor cells are relatively large. This result indicates that it has greater cytotoxicity to both tumor cells, and the cytostatic effect gradually increases with the extension of the culture time [24]. It can be seen from the table that the IC50 value that acts on lung tumor cells is

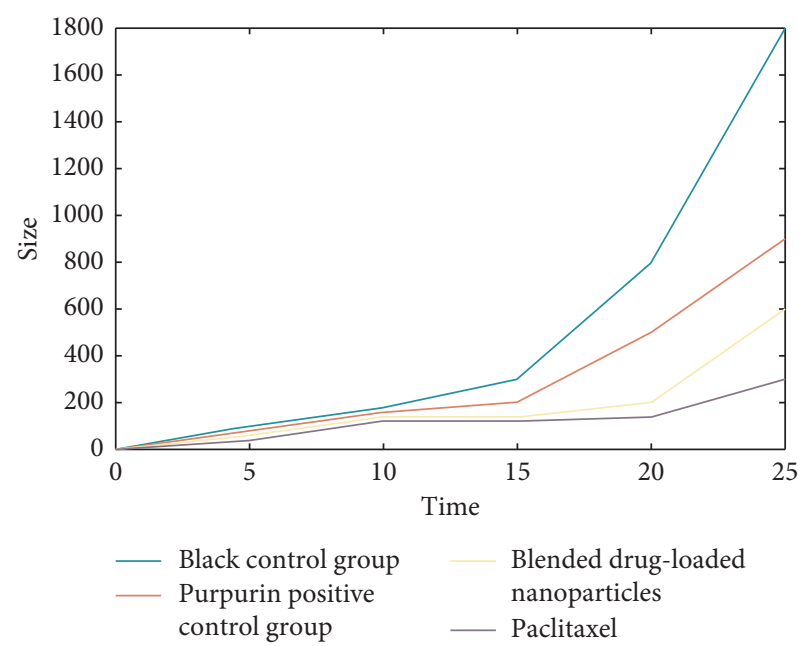

FIGURE 3: Line chart of lung tumor volume change.

TABLE 1: IC50 values of lung tumor cells.

\begin{tabular}{lccc}
\hline \multirow{2}{*}{ Drug } & IC50 $(\mu \mathrm{g} / \mathrm{ml})$ & \\
& Time $(\mathrm{h})$ & 1.22 & MCF-7 \\
\hline \multirow{3}{*}{ PTX } & 24 & 0.84 & 0.8 \\
& 48 & 0.77 & 0.77 \\
& 72 & 1.85 & 0.33 \\
\hline \multirow{3}{*}{ HA } & 24 & 1.03 & 1.49 \\
& 48 & 0.93 & 0.5 \\
& 72 & & 0.33 \\
\hline
\end{tabular}

smaller than that of lung tumor cells. The drug acts on different tumor cells. The preparation group has a better choice for MCF-7 lung tumor cells with high HA receptor expression. It can be seen from the table that the IC50 value that acts on lung tumor cells is smaller than that of lung tumor cells. The drug acts on different tumor cells, the preparation group has better selectivity and higher cytotoxicity for MCF-7 lung tumor cells with high HA receptor expression. With the extension of the culture time, the inhibitory effect of the preparation HA group on HepG2 cells was similar to that of the PTX group, while the inhibitory effect on lung tumor MCF-7 cells was stronger than that of the PTX group. The results show that the preparation HA can well inhibit the growth of tumor cells and has a significant effect on tumor cells with high expression of CD44 receptor.

The positive control is a drug group containing the same concentration of free paclitaxel, and the negative control is a blank medium without anticancer drugs. The survival rate of the cells in the negative control group is calculated as $100 \%$. The killing effects of nanoparticles and positive control on lung tumor cells are shown in Figure 4, respectively. The results showed that the killing effect of nanoparticles on lung tumor cells was significantly higher than that of the positive control. The concentration of the added drug was $0.001,0.01$, $0.1,1$, and $10 \mu \mathrm{M}$ in terms of the concentration of paclitaxel. The median survival concentration is lower than that of free paclitaxel [25]. 


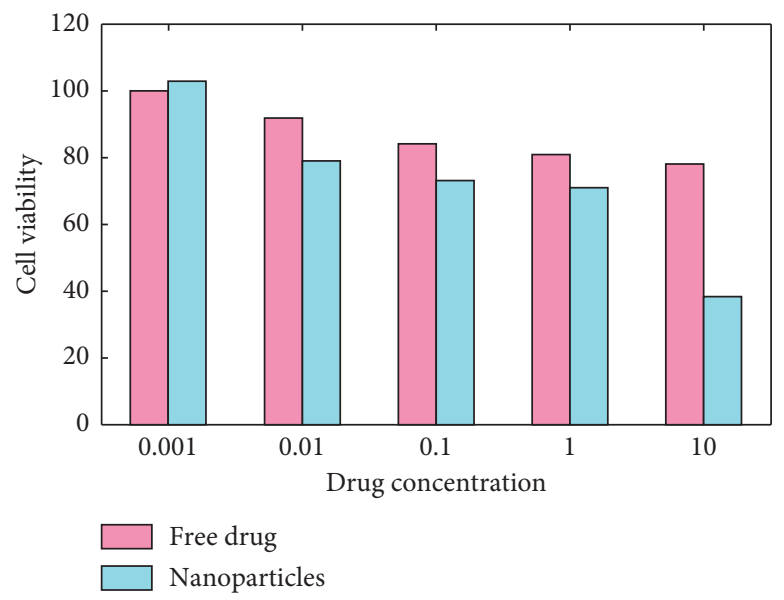

Figure 4: The killing of lung tumor cells by drugs.

It can be seen from the above results that the particle morphology of the nanoparticles provided in this article are relatively uniform and easy to control. The results of the cytotoxicity test showed that for HT29 cells and CT26 cells, the half-survival concentration of the nanoparticles in this article was lower than the free paclitaxel drug, which indicated that the nanoparticles had a highly effective killing effect on HT29 cells and CT26 cells. Therefore, the nanoparticles containing anticancer drugs provided in this article can enter tumor cells more efficiently and kill them, and the effect is better than that of free drugs [26].

\subsection{Analysis of Motion Flow in Tennis Rehabilitation Training.} The upper limb dyskinesia of patients is mainly reflected in the limited range of motion when completing related actions. According to the evaluation method mentioned above to determine the degree of rehabilitation, this article divides the evaluation results of the motion flow similarity of each group of training actions into five levels. Due to individual differences, the range of joint motion given in the article is based on the percentage of the maximum joint motion of the patient's healthy limb. Table 2 shows the fivelevel classification table of shoulder joint-abduction movements [27].

The somatosensory rehabilitation training system designed in this paper captures and calculates the real-time angle and angular velocity of the corresponding joints while capturing the three-dimensional motion coordinates of the human joint points [28]. As above, this article chooses the maximum joint angle value that the patient can achieve in a set of training process as the patient's joint range of motion and calculates the waveform similarity of the template action and the training action joint angle waveform. The calculation result is used as the scoring result of the completion of the training action. This set of experiments selects the joint angle information corresponding to the five sets of training data in Table 2. Taking the fifth set of experimental data as an example, draw a comparison chart of the training action shoulder joint angle curve and the fifth-level template joint angle curve in MATLAB, as shown in Figure 5.
TABLE 2: Five-level classification of abduction movements.

\begin{tabular}{lcccc}
\hline \multirow{2}{*}{$\begin{array}{l}\text { Training } \\
\text { action }\end{array}$} & $\begin{array}{c}\text { Action } \\
\text { level }\end{array}$ & $\begin{array}{c}\text { Doint range } \\
\text { of motion }\end{array}$ & $\begin{array}{c}\text { Scope of this } \\
\text { article }\end{array}$ & Rating \\
\hline & & $0 \% \sim 50 \%$ & $0^{\circ} \sim 70^{\circ}$ & $\begin{array}{c}\text { Level } \\
1\end{array}$ \\
& Insufficient & & & $\begin{array}{c}\text { Level } \\
2\end{array}$ \\
$\begin{array}{l}\text { Shoulder } \\
\text { abduction }\end{array}$ & & $60 \% \sim-65 \%$ & $70^{\circ} \sim-85^{\circ}$ & $\begin{array}{c}\text { Level } \\
3\end{array}$ \\
& & $65 \% \sim 75 \%$ & $85^{\circ} \sim 125^{\circ}$ & $\begin{array}{c}3 \\
\text { Level } \\
4\end{array}$ \\
& Full & $75 \% \sim 90 \%$ & $125 \% \sim 145^{\circ}$ & $\begin{array}{c}4 \\
\text { Level } \\
5\end{array}$ \\
\hline
\end{tabular}

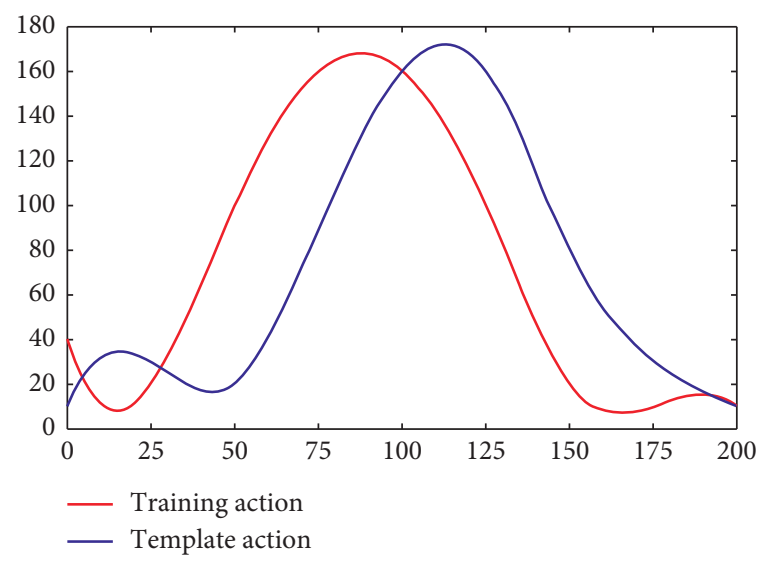

Figure 5: Comparison of joint angles.

From the data analysis in the figure, it can be concluded that the maximum angular velocity of the two trainings is the same, and the overall upward or downward trend is the same. The maximum angular velocity is 170 . It can be seen from the training movement and the fourth-level template movement trajectory. The degree of coincidence is the highest; that is, the training rating is judged to be the fourth 
level. This is the same as the result obtained by applying the fusion algorithm; that is, it is feasible to apply two distance measure fusion algorithms to determine the similarity of the motion flow [29].

4.4. Analysis of the Treatment of Lung Tumors by Sports Rehabilitation Training. Sports rehabilitation training played a role both during and after the tumor treatment. The results of the study showed that, during the treatment of breast cancer survivors, the experimental group achieved significant improvements in function, mental health, and main complaints and after the treatment in oxygen demand, changes in cardiopulmonary function, self-esteem, depression, and anxiety. There were significant differences among breast cancer survivors in the experimental group.

In patients with peripheral blood stem cell transplantation, exercise rehabilitation guidance has played a positive and beneficial role in certain aspects, such as improving physical fitness (such as non-fatty matter), improving muscle strength and function, improving symptoms (such as pain and diarrhea), shortening hospital stay, and improving mental health and emotional conditions. Sports rehabilitation guidance has also shown many advantages in the treatment of patients with mixed solid tumors, such as physical fitness, aerobic metabolism, bone salt deposition, shoulder mobility, pain, fatigue, completion rate of training programs, and own survival quality and emotional state. Kirshbaum reported that the role of aerobic exercise in breast cancer patients receiving adjuvant therapy was significantly higher than its role after the end of treatment [30].

Sports rehabilitation training can reduce tumor recurrence and prolong overall survival. This article explores the correlation between exercise intervention and survival in a group of female patients with invasive lung tumors. The study followed up 4,000 breast cancer survivors aged 22-77. The longest follow-up time among the participants was 5 years. Women who participated in more exercise had lower breast cancer mortality and all-cause mortality (38\% to $48 \%)$. Even if the known prognostic factors are adjusted, such as age, family history, disease stage, hormone therapy, treatment method, energy intake, and BMI, its advantages are still significant. Analyzing the correlation of 2000 women's exercise, cancer recurrence, and total mortality in women with early breast cancer through data analysis, it is found that moderate-intensity exercise can reduce mortality, but high-intensity exercise does not.

\section{Conclusions}

The standard matching algorithm discussed in this article has certain practical value for the classification and recognition of actions, but it should be noted that the execution of the algorithm has a long delay; that is, the execution speed of the matching algorithm needs to be improved. In the following research, we will find the optimization strategy of the fusion algorithm and realize the possibility of merging with the distributed computer and other parallel computing methods to reduce the total load time of the system platform.
The application of the nanoparticle carrier technology studied in this paper in the treatment of tumors by PDT not only makes up for the current deficiency of PDT tumor treatment but also adds many new features of PDT, further improving the effectiveness of PDT, reducing adverse effects, and expanding the scope of application of PDT. The adverse effects have expanded the scope of application of PDT. This basic research has fully demonstrated the great potential of nanotechnology in PDT tumor treatment, but how to transform this technology from laboratory research to industrial production is a big problem. Nanoparticle carriers have been used for improvement many times. But how to transform this technology from laboratory research to industrial production is a big problem. Nanoparticle carriers have been used many times to improve the functions of various organs in the human body. Its long-term impact remains to be seen. Its long-term effects remain to be seen.

In this paper, Camel was used as a template to design and screen new high-performance paclitaxel nanoparticles with anticancer activity. Based on this, we will do the following work on peptide carriers: (1) develop targeted anticancer drugs through receptor-mediated targeting molecules and chimera 18-Camel; (2) coordinate anticancer drugs by combining targeted drugs and anticancer drugs with siRNA carrying tumor suppressor genes and antirejection genes; (3) to study the anticancer effect in mice, the synergistic anticancer effect in vivo as a gene carrier, the distribution of paclitaxel in mice, and the evaluation of toxicity.

\section{Data Availability}

No data were used to support this study.

\section{Conflicts of Interest}

The authors declare that they have no conflicts of interest regarding the publication of the research article.

\section{References}

[1] F. Noveletto, F. L. F. Soares, S. C. Domenech, M. d. S. Hounsell, and P. B. Filho, "Biomedical serious game system for lower limb motor rehabilitation of hemiparetic stroke patients," IEEE Transactions on Neural Systems and Rehabilitation Engineering, vol. 28, no. 6, pp. 1481-1487, 2020.

[2] Y. Yu, S. Xu, H. You et al., "In vivo synergistic anti-tumor effect of paclitaxel nanoparticles combined with radiotherapy on human cervical carcinoma," Drug Delivery, vol. 24, no. 1, pp. 75-82, 2017.

[3] T. Rozario, T. D. Chiu, M. Chen et al., "A novel markerless lung tumor-tracking method using treatment $\mathrm{MV}$ beam imaging," Applied Sciences, vol. 8, no. 12, p. 2525, 2018.

[4] S. Radu-Tiberiu and B. Alin Marius, "Improving the quality of life through participation in physical activity- the tennis camp pilot study," Timisoara Physical Education and Rehabilitation Journal, vol. 10, no. 19, pp. 159-164, 2017.

[5] P.-C. Leung, "Rehabilitation training in artificially heated environment," Journal of Exercise Rehabilitation, vol. 13, no. 5, pp. 546-549, 2017.

[6] C. M. Custodio and E. M. Wisotzky, "Is cancer rehabilitation fellowship training necessary for graduating physiatrists who 
wish to enter the field of cancer rehabilitation?" $P M \& R$ the Journal of Injury Function \& Rehabilitation, vol. 9, no. 9, p. S429, 2017.

[7] N. Geoffroy, C. Michel, M. Bernard, and P. Jacques, "Os acromiale in professional tennis players," Orthopaedic Journal of Sports Medicine, vol. 6, no. 5, Article ID 232596711877372, 2018.

[8] N. Li, H. Cai, L. Jiang et al., "Enzyme-sensitive and amphiphilic PEGylated dendrimer-paclitaxel prodrug-based nanoparticles for enhanced stability and anticancer efficacy," ACS Applied Materials \& Interfaces, vol. 9, no. 8, pp. 6865-6877, 2017.

[9] T. Hu, C. Qian, Y. Xuan et al., "Radionuclide I-131 labeled albumin-paclitaxel nanoparticles for synergistic combined chemo-radioisotope therapy of cancer," Theranostics, vol. 7, no. 3, pp. 614-623, 2017.

[10] S. Tiwari, A. B. Tirosh, and A. Rubinstein, "Increasing the affinity of cationized polyacrylamide-paclitaxel nanoparticles towards colon cancer cells by a surface recognition peptide," International Journal of Pharmaceutics, vol. 531, no. 1, pp. 281-291, 2017.

[11] F. Xie, W.-F. Ding, Z.-J.-L. Liu et al., "In vivo antitumor effect of endostatin-loaded chitosan nanoparticles combined with paclitaxel on Lewis lung carcinoma," Drug Delivery, vol. 24, no. 1, pp. 1410-1418, 2017.

[12] L. N. Turino, M. R. Ruggiero, R. Stefanìa, J. C. Cutrin, S. Aime, and S. Geninatti Crich, "Ferritin decorated PLGA/paclitaxel loaded nanoparticles endowed with an enhanced toxicity toward MCF-7 breast tumor cells," Bioconjugate Chemistry, vol. 28, no. 4, pp. 1283-1290, 2017.

[13] V. Elena, B. Chiara, C. Alessia et al., "Albumin and hyaluronic acid-coated superparamagnetic iron oxide nanoparticles loaded with paclitaxel for biomedical applications," Molecules, vol. 22, no. 7, p. 1030, 2017.

[14] T. Wang, Y. Liu, and C. Wu, "Effect of paclitaxel-mesoporous silica nanoparticles with a core-shell structure on the human lung cancer cell line A549," Nanoscale Research Letters, vol. 12 , no. 1 , p. $66,2017$.

[15] T. M. Celik, Y. Çiğdem, U. Sedat et al., "Development and characterization of paclitaxel-loaded PLGA nanoparticles and evaluation of cytotoxicity on MCF-7 cell line by MTT assay," Proceedings, vol. 1, no. 10, p. 1030, 2017.

[16] F. Madani, B. Esnaashari, B. Mujokoro, F. Dorkoosh, M. Khosravani, and M. Adabi, "Investigation of effective parameters on size of paclitaxel loaded PLGA nanoparticles," Advanced Pharmaceutical Bulletin, vol. 8, no. 1, pp. 77-84, 2018.

[17] K. J. Surendra, T. Suchi, J. Ruchi, and J. Nilesh, "Formulation and evaluation of protein bound paclitaxel nanoparticles for injectable suspension," Journal of Drug Delivery and Therapeutics, vol. 10, no. 3, pp. 51-57, 2020.

[18] J. Li, Y. Gu, Z. Wei et al., "Molecular mechanism for selective cytotoxicity towards cancer cells of diselenide-containing paclitaxel nanoparticles," International Journal of Biological Sciences, vol. 15, no. 8, pp. 1755-1770, 2019.

[19] N. Yu, J. Li, Z. Yuan, D. Dan, L. Xiaolin, and X. Huae, "Superior antitumor effect of self-assembly supramolecular paclitaxel nanoparticles," RSC Advances, vol. 10, no. 22, pp. 12999-13005, 2020.

[20] Z. Min, L. Xin, L. Yuanyuan et al., "Ascorbyl palmitate-incorporated paclitaxel-loaded composite nanoparticles for synergistic anti-tumoral therapy," Drug Delivery, vol. 24, no. 1, pp. 1230-1242, 2017.
[21] J. Mo, L. Wang, X. Huang et al., "Multifunctional nanoparticles for co-delivery of paclitaxel and carboplatin against ovarian cancer by inactivating the JMJD3-HER2 axis," Nanoscale, vol. 9, no. 35, p. 13142, 2017.

[22] L. F. Wei, "Comparison between corticosteroid and lidocaine injection in the treatment of tennis elbow: a randomized, double-blinded, controlled trial," American Journal of Physical Medicine \& Rehabilitation, vol. 97, no. 9, p. 1, 2018.

[23] P. C. Chao, T. C. Yeh, C. L. Huang et al., "Successful treatment of bipolar disorder by resection of lung tumor: a case report," Indian Journal of Psychiatry, vol. 60, no. 3, pp. 369-370, 2018.

[24] H. Mamdani, S. Ahmed, S. Armstrong, M. Tony, and I. J. Shadia, "Blood-based tumor biomarkers in lung cancer for detection and treatment," Translational Lung Cancer Research, vol. 6, no. 6, pp. 648-660, 2017.

[25] S.-W. Kim, Y. K. Lee, J. H. Hong et al., "Cancer treatment: mutual destruction of deep lung tumor tissues by nanodrugconjugated stealth mesenchymal stem cells (adv. Sci. 5/2018)," Advanced Science, vol. 5, no. 5, p. 1870030, 2018.

[26] X. Zhu, L.-Z. Zhu, and R.-J. Yang, "Role of inferior phrenic artery in the interventional treatment of lung metastases tumor: a report of 11 cases," Journal of Cancer Research and Therapeutics, vol. 14, no. 1, p. 61, 2018.

[27] J. Miyamoto, T. Nakagawa, and H. Hirayama, "Metastatic brain tumor from lung adenocarcinoma presenting a unique radiographic pattern during afatinib treatment: a case report," No Shink Geka Neurological Surgery, vol. 46, no. 3, p. 213, 2018.

[28] M. Jiang, N. Zhou, D. Liu et al., "P3.03-018 tumor cavitation in lung metastases in patients with solid tumor treated with apatinib," Journal of Thoracic Oncology, vol. 12, no. 11, p. S2280, 2017.

[29] Y. Peng, Y. Wang, and X. Z. Hao, "Utility of multiple increased lung cancer tumor markers in treatment of patients with advanced lung adenocarcinoma," Zhongguo Fei Ai $\mathrm{Za}$ Zhi/Chinese Journal of Lung Cancer, vol. 20, no. 10, pp. 690-694, 2017.

[30] M. Nagasaka, M. Crosby, N. Thummala, and A. J. SukariAbrams, "P2.07-040 pre-treatment tumor volume in non-small cell lung cancer (NSCLC) as a predictor of response to PD-1 inhibitors," Journal of Thoracic Oncology, vol. 12, no. 11, p. S2431, 2017. 